\title{
Can we avoid high levels of dose escalation for high-risk prostate cancer in the setting of androgen deprivation?
}

This article was published in the following Dove Press journal:

OncoTargets and Therapy

II May 2016

Number of times this article has been viewed

\author{
Thomas P Shakespeare ${ }^{1,2}$ \\ Shea W Wilcox' \\ Noel J Aherne ${ }^{1,2}$ \\ 'Department of Radiation Oncology, \\ North Coast Cancer Institute, ${ }^{2}$ Rural \\ Clinical School, Faculty of Medicine, \\ University of New South Wales, Coffs \\ Harbour, NSW, Australia
}

Aim: Both dose-escalated external beam radiotherapy (DE-EBRT) and androgen deprivation therapy (ADT) improve outcomes in patients with high-risk prostate cancer. However, there is little evidence specifically evaluating DE-EBRT for patients with high-risk prostate cancer receiving ADT, particularly for EBRT doses $>74 \mathrm{~Gy}$. We aimed to determine whether DEEBRT $>74$ Gy improves outcomes for patients with high-risk prostate cancer receiving longterm ADT.

Patients and methods: Patients with high-risk prostate cancer were treated on an institutional protocol prescribing 3-6 months neoadjuvant ADT and DE-EBRT, followed by 2 years of adjuvant ADT. Between 2006 and 2012, EBRT doses were escalated from 74 Gy to 76 Gy and then to $78 \mathrm{~Gy}$. We interrogated our electronic medical record to identify these patients and analyzed our results by comparing dose levels.

Results: In all, 479 patients were treated with a 68 -month median follow-up. The 5-year biochemical disease-free survivals for the $74 \mathrm{~Gy}, 76 \mathrm{~Gy}$, and $78 \mathrm{~Gy}$ groups were $87.8 \%, 86.9 \%$, and $91.6 \%$, respectively. The metastasis-free survivals were $95.5 \%, 94.5 \%$, and $93.9 \%$, respectively, and the prostate cancer-specific survivals were $100 \%, 94.4 \%$, and $98.1 \%$, respectively. Dose escalation had no impact on any outcome in either univariate or multivariate analysis.

Conclusion: There was no benefit of DE-EBRT $>74$ Gy in our cohort of high-risk prostate patients treated with long-term ADT. As dose escalation has higher risks of radiotherapyinduced toxicity, it may be feasible to omit dose escalation beyond 74 Gy in this group of patients. Randomized studies evaluating dose escalation for high-risk patients receiving ADT should be considered.

Keywords: radiotherapy, IMRT, dose, dose escalation, dose de-escalation, androgen deprivation therapy, prostate cancer

\section{Introduction}

Both dose-escalated external beam radiotherapy $(\mathrm{DE}-\mathrm{EBRT})^{1}$ and androgen deprivation therapy (ADT) combined with radiotherapy ${ }^{2}$ have been shown to improve prostate cancer outcomes based on meta-analyses of randomized trials. It is also thought that in the absence of ADT, EBRT doses $>70$ Gy provide a benefit, ${ }^{3-6}$ with several national guidelines recommending DE-EBRT up to 78-81 Gy for high-risk patients. ${ }^{7-9}$ These guidelines also recommend consideration of ADT with DE-EBRT. However, it has been noted that there is virtually no evidence for dose-escalating EBRT to these high dose levels in the setting of ADT. ${ }^{10}$

There are only two randomized trials of DE-EBRT for patients receiving ADT. ${ }^{11,12}$ Both compare 64 Gy EBRT with 74 Gy DE-EBRT. In these studies, all patients received 
neoadjuvant ADT. Only one reported a significant benefit for dose escalation up to $74 \mathrm{~Gy}$ in the high-risk group. ${ }^{11}$ The lack of data in general and the specific paucity for patients receiving $>74$ Gy has led some to question the benefit of DE-EBRT for patients receiving ADT. ${ }^{10,13}$

Given the lack of available evidence, we evaluated whether dose escalation up to 78 Gy had any advantage in terms of prostate-specific antigen (PSA) disease-free, metastasis-free, or prostate cancer-specific survival (PCaSS) outcomes in our cohort of high-risk patients treated exclusively with long-term ADT and dose-escalated intensitymodulated radiotherapy (IMRT).

\section{Patients and methods}

Since 2006, the North Coast Cancer Institute has been implementing a dose-escalation program for patients with localized prostate cancer. The patients were initially treated with a dose of 73.8-74 Gy, which was escalated to 76 Gy in 2008 and then 78 Gy from 2009 until 2012. All patients were treated with either three-dimensional EBRT or IMRT, as has been previously reported. ${ }^{14,15}$ In brief, after institutional ethics approval (North Coast New South Wales Human Research Ethics Committee, reference number QA 101), the electronic medical record of our institution (Mosaiq; Elekta, Crawley, UK) was interrogated to identify all patients with National Comprehensive Cancer Network-defined high-risk prostate cancer $^{9}$ treated with our standard protocol of DE-EBRT and ADT. The exclusion criteria included patients post prostatectomy, had NCCN-defined low or high risk, were node positive, were metastatic, had histology other than adenocarcinoma, did not receive ADT, or were treated from 2013 onward. Patient consent was not required as this was a retrospective study

All patients were staged prior to treatment with computed tomography (CT) of the abdomen and pelvis and whole body nuclear medicine bone scan. The patients were prescribed ADT using leuprorelin or goserelin acetate monotherapy (using 3-4-month depots), with 3-6 months of neoadjuvant/ concurrent ADT and 2 years of adjuvant ADT. The patients underwent transrectal ultrasound-guided insertion of fiducial markers followed by magnetic resonance imaging/CT fusion as previously reported, ${ }^{16}$ unless contraindicated. The patients were treated on our "Bowel and Bladder Protocol" involving low-residue diet, aperients, and a pretreatment oral fluid regimen to achieve a comfortably full bladder and empty rectum. The planning CT ( $2 \mathrm{~mm}$ slices) was performed with patients lying supine and immobilized with ankle stocks. The patients with seminal vesicle (SV) invasion (either clinically or on MRI) had the prostate and SVs included in the full-dose clinical target volume (CTV), treated to $73.8-78$ Gy in
1.8-2.0 Gy fractions prescribed to the reference point. The patients without SV invasion had the prostate and proximal 4-8 mm SVs included in the full-dose CTV, and the distal SVs were included in a lower dose CTV (50 Gy, equivalently treated via an integrated boost). The CTV to planning target volume expansion was $5 \mathrm{~mm}$. The patients were treated using a seven- to nine-field IMRT technique with the angles optimized to achieve target coverage and organ at risk sparing. Image guidance utilized either daily online kilovoltage portal images (matched to fiducial markers) or daily cone beam CT. The patients without fiducial markers $(<1 \%$ of all patients) underwent daily cone beam CT matching to soft tissue and bone. The patients were followed up by the treating radiation oncologists, with data prospectively recorded in Mosaiq. Biochemical failure was determined using the Phoenix definition (PSA nadir $+2 \mathrm{ng} / \mathrm{mL}$ ). All patients with biochemical failure were restaged with $\mathrm{CT}$ and bone scans, and more recently, prostate-specific membrane antigen positron emission tomography. ${ }^{17}$ Salvage ADT was initiated when the PSA reached 10-20 ng/mL or with documented metastatic disease.

Data were analyzed using SPSS v19 (IBM Corporation, Armonk, NY, USA). The 73.8 Gy and 74 Gy groups were combined into a single group designated as 74 Gy. Biochemical disease-free survival (bDFS; which we define as survival free of the event of PSA relapse of prostate cancer), metastasis-free survival (MFS; which we define as survival free of the event of metastatic relapse of prostate cancer), and PCaSS were calculated using Kaplan-Meier curves, and the log-rank (Mantel-Cox) test was used to compare survival between groups. The follow-up time was calculated from the date of commencement of ADT as recommended by Denham et al. ${ }^{18}$ Univariate analysis was done to assess the relationship between potential prognostic factors and bDFS, MFS, and PCaSS. The variables included were age $(\leq 70$ vs $>70)$, pretreatment PSA $(<10 \mathrm{ng} / \mathrm{mL}$ vs $\geq 10 \mathrm{ng} / \mathrm{mL}$ ), Gleason score (6-7 vs $8-10)$, use of IMRT (no vs yes), and radiation dose (74 Gy, 76 Gy, and $78 \mathrm{~Gy})$. The variables with a statistical significance $<0.25$ were taken into account in a multivariate Cox regression analysis. All hazard ratios (HRs) were calculated with Cox proportional hazard models and expressed relative to the control group. $P$-values were two tailed and considered statistically significant if $<0.05$.

\section{Results}

In total, there were 479 high-risk patients treated with DEEBRT and ADT, with a median follow-up of 68 months. Patient demographics are shown in Table 1. The 5-year 


\section{Table I Patient characteristics}

\begin{tabular}{ll}
\hline Age, years & 72 \\
Median & $47-85$ \\
Range & \\
Gleason score, $n$ (\%) & $151(31.5)$ \\
$6-7$ & $328(68.5)$ \\
$8-10$ & \\
IMRT, n (\%) & $13(2.7)$ \\
No & $466(97.3)$ \\
Yes & \\
Dose, $n(\%)$ & $96(20.0)$ \\
74 Gy & $56(11.7)$ \\
76 Gy & $327(68.3)$ \\
78 Gy & \\
PSA, ng/mL & 13.7 \\
$\quad$ Median & $1.2-180.0$ \\
Range & \\
Tumor stage, n (\%) & $299(62.4)$ \\
TI-2 & $180(37.6)$ \\
$\quad$ T3-4 & \\
\hline Abbreviations: IMRT, intensity-modulated radiotherapy; PSA, prostate-specific antigen.
\end{tabular}

bDFS for all 479 patients was $90.0 \%$. The bDFS for 74 Gy, $76 \mathrm{~Gy}$, and 78 Gy groups, respectively, were $87.8 \%, 86.9 \%$, and 91.6\% (Kaplan-Meier curves shown in Figure 1). These differences were not statistically significant $(P=0.8)$.

The dose level had no significant effect on bDFS in either uni- or multivariate analysis. In multivariate analysis, factors predicting a higher hazard for biochemical failure included T3-4 disease (HR 2.0, 95\% CI 1.2-3.5, $P=0.009$ ), PSA $>10$ (HR 2.5, 95\% CI 1.3-4.7, $P=0.006$ ), and Gleason scores 8-10 (HR 2.9, 95\% CI 1.5-5.9, $P=0.003$ ). Further analyses grouping $74-76$ Gy vs 78 Gy and 74 Gy vs $76-78$ Gy also showed no effect on bDFS.

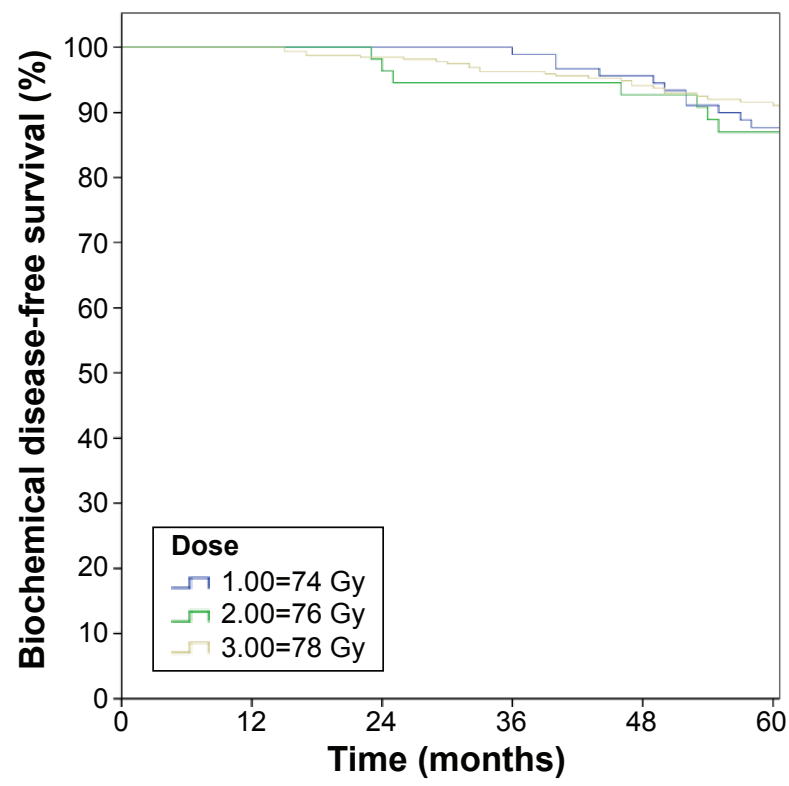

Figure I Biochemical disease-free survival for various dose levels.

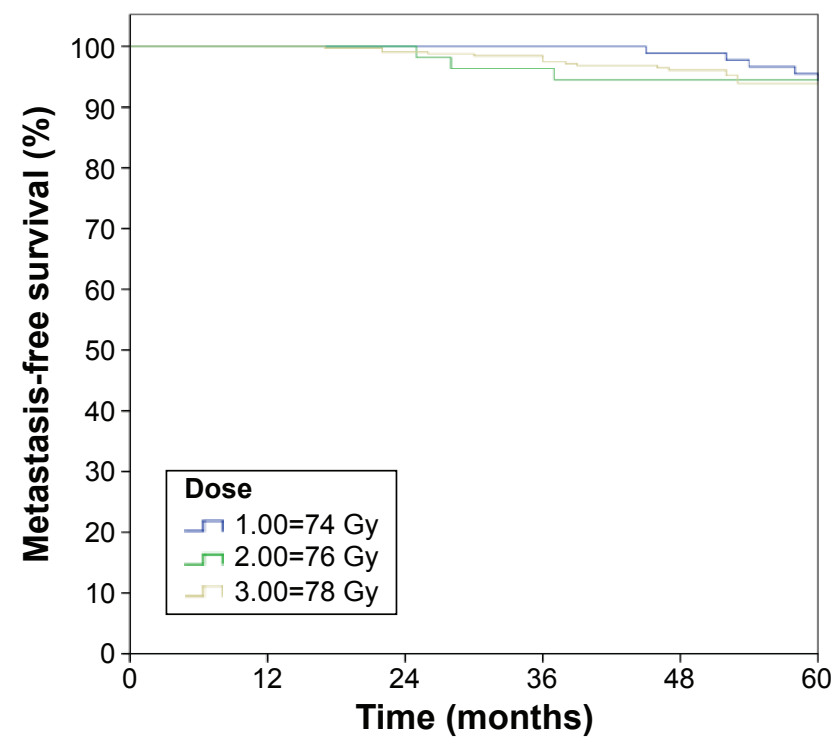

Figure 2 Metastasis-free survival for various dose levels.

The 5-year MFS was $94.3 \%$. The MFS for $74 \mathrm{~Gy}, 76 \mathrm{~Gy}$, and 78 Gy groups, respectively, were $95.5 \%, 94.5 \%$, and 93.9\% (MFS Kaplan-Meier curves shown in Figure 2). The dose level had no impact on MFS ( $P=0.8)$. Factors predicting a higher hazard of metastatic failure in multivariate analysis included T3-4 (HR 2.1, 95\% CI 1.0-3.8, $P=0.048$ ) and Gleason scores 8-10 (HR 2.9, 95\% CI 1.2-7.1, $P=0.02$ ).

The 5-year PCaSS was $98.1 \%$. The PCaSS for $74 \mathrm{~Gy}$, $76 \mathrm{~Gy}$, and 78 Gy groups, respectively, were $100 \%, 94.4 \%$, and $98.1 \%$ (Kaplan-Meier curves shown in Figure 3). The dose level had no impact on MFS $(P=0.6)$, and neither did any other factor.

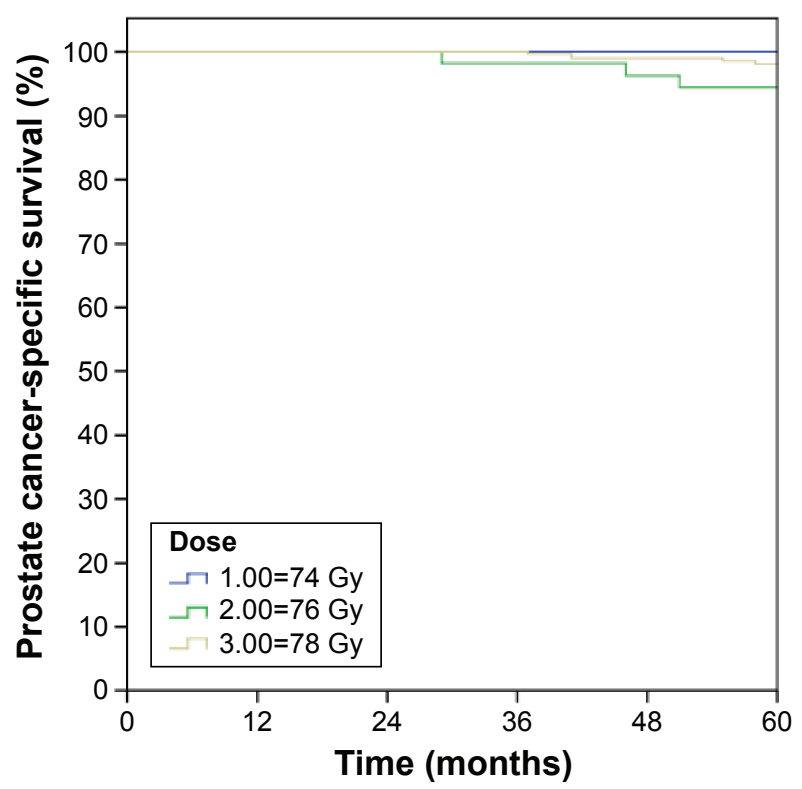

Figure 3 Prostate cancer-specific survival for various dose levels. 


\section{Discussion}

Dose escalation has been shown to improve bDFS for patients with localized prostate cancer; ${ }^{1}$ however, ADT, particularly for high-risk disease, not only improves bDFS but also PCaSS and the overall survival. ${ }^{2,19}$ Not surprisingly, questions have been raised about the need for dose escalation in patients receiving ADT. ${ }^{10,13}$ There are two randomized trials that have investigated this dilemma. ${ }^{11,12}$ Creak et al, in a randomized Phase II trial, found a nonstatistically significant trend for PSA control favoring 74 Gy over 64 Gy. The reason for the nonsignificant result cannot be ascertained; perhaps, there was truly no difference between the groups or there were insufficient patient numbers. Regardless, Creak et $\mathrm{al}^{12} \mathrm{did}$ not analyze the results for high-risk patients. On the other hand, Dearnaley et al, in a randomized Phase III trial, found a significant progression-free survival benefit of DE-EBRT for 74 Gy (compared to 64 Gy). This finding was also significant for the high-risk subgroup. ${ }^{11}$ However, this study is limited by the fact that EBRT doses were only escalated to 74 Gy and that patients only received short-course ADT (whereas long-term ADT has been found to be superior in some studies). ${ }^{20}$

A few other studies evaluating dose escalation in the setting of ADT did not analyze results for high-risk patients and EBRT doses $>74$ Gy. For example, Denham et $\mathrm{al}^{20}$ conducted a nonrandomized evaluation of EBRT dose levels up to 74 Gy and 46 Gy EBRT combined with high-dose rate brachytherapy. They found that higher doses did reduce local progression; however, there was no analysis of bDFS. In their study, there were 844 high-risk patients; however, the results were not analyzed for this group.

A study by Stoyanova et $\mathrm{al}^{13}$ evaluated the relative benefits of ADT and DE-EBRT up to $80 \mathrm{~Gy}$; however, the results were not reported by the risk group. Despite this, the authors were able to conclude that the benefit of ADT far outweighed the benefit of dose escalation. This belief was echoed by Roach, ${ }^{10}$ who also attempted to address the relative benefits of dose escalation vs ADT via a literature review. With a paucity of high-quality studies, he concluded that the data supporting ADT were greater than the data for dose escalation. Indeed, in a previous series of patients with intermediate risk prostate cancer, we were unable to find any benefit of dose escalation in the setting of neoadjuvant ADT. ${ }^{21}$

In the present series, we report one of the largest cohort of patients with high-risk prostate cancer treated exclusively with a combination of long-term ADT and DE-EBRT. As far as we are aware, this is the only study evaluating the potential benefit of dose escalation beyond 74 Gy in the setting of long-term (2-3 years) ADT and EBRT in the management of this risk group. We failed to demonstrate any statistically or clinically significant benefit for dose escalation up to $78 \mathrm{~Gy}$.

Although dose escalation may have questionable benefits in terms of cancer outcomes, the adverse effects are well known. A meta-analysis of randomized trials of dose escalation with long-term follow-up showed that dose escalation increases both late genitourinary and gastrointestinal toxicities. ${ }^{1}$ Even limited dose escalation from $74 \mathrm{~Gy}$ to $78 \mathrm{~Gy}$ in 2 Gy fractions has been shown to significantly increase toxicity. ${ }^{22}$ It is known that ADT has its own toxicities; however, many of these toxicities are potentially reversible. ${ }^{23}$ Additionally, in some studies, ADT has been found to protect against both late radiotherapy-induced urinary and bowel toxicities, ${ }^{23,24}$ perhaps partly due to a reduction in prostate size reducing bladder and rectal dose. ${ }^{25}$

If $\mathrm{ADT}$ is more beneficial than dose escalation, it may be possible to limit dose escalation to 74 Gy or possibly even reduce doses further (eg, to $70 \mathrm{~Gy}$ ). Dose reduction has been possible in other cancer types where systemic therapy is given. ${ }^{26,27}$ It seems evident that further research should evaluate the relative benefits of high radiotherapy doses in the setting of long-term ADT for high-risk prostate cancer.

Our study has several limitations. We report a retrospective review, and thus, our findings should be regarded as hypothesis generating only. Although we have a reasonable median follow-up of 68 months, it is possible that any benefits of dose escalation might only occur with longer follow-up, as seen in the study of Dearnaley et al ${ }^{11}$ of 74 Gy vs 64 Gy (having a 10-year median follow-up). Having said this, Dearnaley et al's biochemical progression-free survival curves started diverging at 2-3 years of follow-up and were separated by 5 years (indeed, nearly as separated as the curves at 10 years). We also do not report on doses $>78 \mathrm{~Gy}$, and it is possible that benefits may only be seen with much higher doses. We also note that prostate-specific membrane antigen positron emission tomography imaging was only available for the most recent follow-up period, ${ }^{17}$ and this may have unknown effects on our outcomes. Finally, we have not reported about toxicity, with the unknown interactions and relative quality of life between escalating doses and ADT requiring further study.

\section{Conclusion}

For the high-risk prostate cancer patients receiving neoadjuvant and adjuvant ADT, we found no benefit of increasing radiotherapy doses from 74 Gy up to 78 Gy. There is 
little evidence overall for dose escalation in the setting of ADT, and it is apparent that further research (in the form of large-scale prospective randomized controlled studies) is required to investigate dose escalation, possible dose deescalation, and the interaction of dose escalation and ADT in terms of toxicity and quality of life.

\section{Author contributions}

TPS participated in the design of the study and data collection, performed the statistical analysis, and helped to draft the manuscript. SWW and NJA participated in data collection and helped to draft the manuscript. All authors read and approved the final manuscript.

\section{Disclosure}

The authors report no conflicts of interest in this work.

\section{References}

1. Hou Z, Li G, Bai S. High dose versus conventional dose in external beam radiotherapy of prostate cancer: a meta-analysis of long-term follow-up. J Cancer Res Clin Oncol. 2015;141(6):1063-1071.

2. Schmidt-Hansen M, Hoskin P, Kirkbride P, Hasler E, Bromham N. Hormone and radiotherapy versus hormone or radiotherapy alone for non-metastatic prostate cancer: a systematic review with meta-analyses. Clin Oncol (R Coll Radiol). 2014;26(10):e21-e46.

3. Heemsbergen WD, Al-Mamgani A, Slot A, Dielwart MF, Lebesque JV. Long-term results of the Dutch randomized prostate cancer trial: impact of dose-escalation on local, biochemical, clinical failure, and survival. Radiother Oncol. 2013;110(1):104-109.

4. Kuban DA, Levy LB, Cheung MR, et al. Long-term failure patterns and survival in a randomized dose-escalation trial for prostate cancer. Who dies of disease? Int J Radiat Oncol Biol Phys. 2011;79(5):1310-1317.

5. Zietman AL, Bae K, Slater JD, et al. Randomized trial comparing conventional-dose with high-dose conformal radiation therapy in earlystage adenocarcinoma of the prostate: long-term results from proton radiation oncology group/American college of radiology 95-09. J Clin Oncol. 2010;28(7):1106-1111.

6. Beckendorf V, Guerif S, Le Prise E, et al. 70 Gy versus 80 Gy in localized prostate cancer: 5-year results of GETUG 06 randomized trial. Int J Radiat Oncol Biol Phys. 2011;80(4):1056-1063.

7. Radiotherapy Dose-Fractionation [webpage on the Internet]. The Royal College of Radiologists Board of Faculty of Clinical Oncology; 2006. Available from: https://www.rcr.ac.uk/sites/default/files/publication/ Dose-Fractionation_Final.pdf. Accessed November 29, 2015.

8. Cancer Institute NSW [webpage on the Internet]. Intermediate Risk Prostate Cancer Protocol. Available from: https://www.eviq.org.au/ Protocol/tabid/66/categoryid/185/id/234/Radiation+Oncology $\% 2 \mathrm{c}+$ Prostate \%2c+Intermediate+Risk\%2c+EBRT\%2c+Definitive.aspx. Accessed November 29, 2015.

9. NCCN [webpage on the Internet]. Clinical Practice Guidelines in Oncology. Version 1.2016; 2015. National Comprehensive Cancer Network. Available from: http://www.nccn.org/professionals/physician_gls/pdf/ prostate.pdf. Accessed November 29, 2015.

10. Roach M. Dose escalated external beam radiotherapy versus neoadjuvant androgen deprivation therapy and conventional dose external beam radiotherapy for clinically localized prostate cancer: do we need both? Strahlenther Onkol. 2007;183(Spec No 2):26-28.
11. Dearnaley DP, Jovic G, Syndikus I, et al. Escalated-dose versus controldose conformal radiotherapy for prostate cancer: long-term results from the MRC RT01 randomised controlled trial. Lancet Oncol. 2014;15(4): 464-473.

12. Creak A, Hall E, Horwich A, et al. Randomised pilot study of dose escalation using conformal radiotherapy in prostate cancer: long-term follow-up. Br J Cancer. 2013;109(3):651-657.

13. Stoyanova R, Pahlajani NH, Egleston BL, et al. The impact of doseescalated radiotherapy plus androgen deprivation for prostate cancer using 2 linked nomograms. Cancer. 2013;119(5):1080-1088.

14. Wilcox SW, Aherne NJ, McLachlan CS, McKay MJ, Last AJ, Shakespeare TP. Is modern external beam radiotherapy with androgen deprivation therapy still a viable alternative for prostate cancer in an era of robotic surgery and brachytherapy: a comparison of Australian series. J Med Imaging Radiat Oncol. 2015;59(1):125-133.

15. Wilcox SW, Aherne NJ, Benjamin LC, et al. Long-term outcomes from dose-escalated image-guided intensity-modulated radiotherapy with androgen deprivation: encouraging results for intermediate- and high-risk prostate cancer. Onco Targets Ther. 2014;7:1519-1523.

16. Horsley PJ, Aherne NJ, Edwards GV, et al. Planning magnetic resonance imaging for prostate cancer intensity-modulated radiation therapy: impact on target volumes, radiotherapy dose and androgen deprivation administration. Asia Pac J Clin Oncol. 2014;11(1):15-21.

17. Shakespeare TP. Effect of prostate-specific membrane antigen positron emission tomography on the decision-making of radiation oncologists. Radiat Oncol. 2015;10(1):233.

18. Denham JW, Steigler A, Kumar M, et al. Measuring time to biochemical failure in the TROG 96.01 trial: when should the clock start ticking? Int J Radiat Oncol Biol Phys. 2009;75(4):1008-1012.

19. Jones CU, Hunt D, McGowan DG, et al. Radiotherapy and short-term androgen deprivation for localized prostate cancer. N Engl J Med. 2011; 365(2):107-118.

20. Denham JW, Steigler A, Joseph D, et al. Radiation dose escalation or longer androgen suppression for locally advanced prostate cancer? Data from the TROG 03.04 RADAR trial. Radiother Oncol. 2015;115(3): 301-307.

21. Shakespeare TP, Wilcox SW, Aherne NJ. Can we avoid dose escalation for intermediate-risk prostate cancer in the setting of short-course neoadjuvant androgen deprivation? Onco Targets Ther. 2016;9:1635-1639.

22. Michalski JM, Bae K, Roach M, et al. Long-term toxicity following 3D conformal radiation therapy for prostate cancer from the RTOG 9406 phase I/II dose escalation study. Int J Radiat Oncol Biol Phys. 2010; 76(1):14-22.

23. Denham J, Steigler A, Lamb DS, et al. Short-term androgen deprivation and radiotherapy for locally advanced prostate cancer: results from the Trans-Tasman Radiation Oncology Group 96.01 randomised controlled trial. Lancet Oncol. 2005;6(11):841-850.

24. Zapatero A, García-Vicente F, Sevillano D, et al. Is hormone therapy a protective factor for late hematuria after high-dose radiotherapy in prostate cancer? Urology. 2008;72(5):1130-1134.

25. Samper PM, López Carrizosa MC, Pérez Casas A, et al. Impact of neoadjuvant hormonal therapy on dose-volume histograms in patients with localized prostate cancer under radical radiation therapy. Clin Transl Oncol. 2006;8(8):599-605.

26. Herskovic A, Martz K, al-Sarraf M, et al. Combined chemotherapy and radiotherapy compared with radiotherapy alone in patients with cancer of the esophagus. N Engl J Med. 1992;326(24):1593-1598.

27. Sieber M, Rüffer U, Josting A, Diehl V. Treatment of Hodgkin's disease: current strategies of the German Hodgkin's Lymphoma Study Group. Ann Oncol. 1999;10(suppl 6):23-29. 


\section{Publish your work in this journal}

OncoTargets and Therapy is an international, peer-reviewed, open access journal focusing on the pathological basis of all cancers, potential targets for therapy and treatment protocols employed to improve the management of cancer patients. The journal also focuses on the impact of management programs and new therapeutic agents and protocols on

patient perspectives such as quality of life, adherence and satisfaction. The manuscript management system is completely online and includes a very quick and fair peer-review system, which is all easy to use. Visit http://www.dovepress.com/testimonials.php to read real quotes from published authors.

Submit your manuscript here: http://www.dovepress.com/oncotargets-and-therapy-journal 